\title{
The Method for Recognizing Recognition Helmet Based On Color and Shape
}

\author{
Geng Zhang ${ }^{1}$, Lei $\mathrm{Lv}^{2}$,LI Dan ${ }^{3}$,Min Zhu ${ }^{2}$ \\ (1. China Electric Power Research Institute, Beijing 100192, China;) \\ (2. State Grid Meishan Electric Power Supply Company, sichuan 620010, China); \\ (3. North China Electric Power University, Beijing 1022061, China.)
}

Keyword: Helmet, Face Location, Color feature, Shape Feature

\begin{abstract}
The helmet is widely used in industrial production, in order to prevent accidents, ensure production safety, the establishment of automatic detection of helmet and alarm system becomes more and more urgent. We use intelligent video recognition technology to realize the recognition of the helmet, by mapping the skin color detection and eyes and mouth to locate the face, then scan the upper part of the face, extract color features and shape features to determine whether the head wear a helmet. The method is based on the substation environment, without deliberately artificial, flexible with all kinds of different focal lengths of the camera to complete the verification, and have a higher pass rate.
\end{abstract}

\section{Introduction}

"State Power Grid Corporation of safety regulations (substations and power plants, electrical parts)" provides that: "Any person who enters the production site (offices, control room, duty and service team except room) should wear a helmet." Helmet wearing detection in the power plant and substation personnel safety testing is an important part of, as the most commonly used in the production site of the cheapest personal protection tools, it has saved countless lives ${ }^{[1]}$. For a long time, workers in the construction area of low prevalence overall quality, their safety awareness is not strong and can not consciously take the initiative to wear helmets, leading to production safety accidents still occur frequently ${ }^{[2-3]}$. If the helmet can be identified through surveillance video directly to the staff to the construction, it can be found not wearing a safety or illegal operation and other phenomena. Through the use of existing construction monitoring equipment, automatic identification method of video identification of safety helmet, enhance the supervision level of information in reducing regulatory costs at the same time.

\section{Helmet Recognition Principle}

Due to a weak sense of security, people can not wear a helmet initiative, resulting in production safety accidents still occur frequently, so the majority of production sites have been installed monitoring equipment, Through image processing techniques to identify whether workers wear the helmet, it has important practical significance to ensure the construction safety. Helmet recognition process diagram as shown in figure 1: 


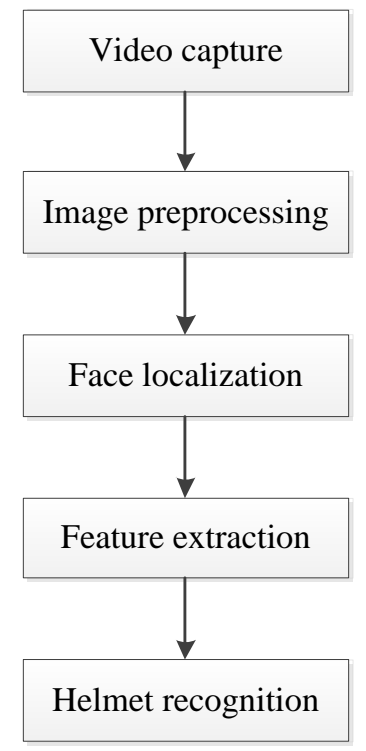

Figure 1. Helmet recognition process

\section{Face localization}

Compared to other more mature human biometric identification methods, such as fingerprints, DNA detection, human face image is hidden and easy to obtain, especially in the non contact environment and is not to disturb the detection of human cases, the superiority of face recognition is far more than other identification methods ${ }^{[4]}$. We use the skin color model to identify the face region, use eyes and mouth triangular face ellipse template and face matching template, eventually to locate human faces.

In order to eliminate the influence of illumination factor, we color the input image using $\mathrm{YCbCr}$ color model for color space conversion, the conversion from color components associated with high RGB color components of relatively small space to $\mathrm{YCbCr}$ color space, color component of the correlation of RGB and YCbCr conversion formula is:

$$
\left[\begin{array}{c}
Y \\
\mathrm{Cb} \\
\mathrm{Cr} \\
1
\end{array}\right]=\left[\begin{array}{cccc}
0.2990 & 0.5870 & 0.1140 & 0 \\
-0.1687 & -0.3313 & 0.5000 & 128 \\
0.5000 & -0.4187 & -0.0813 & 128 \\
0 & 0 & 0 & 1
\end{array}\right]\left[\begin{array}{l}
R \\
G \\
B \\
1
\end{array}\right]
$$

Considering the color clustering region is along with the changes of $\mathrm{Y}$ and presents a piecewise linear change, before skin color detection, the image is segmented by linear color transformation:

$$
C_{i}(Y)=\left\{\begin{array}{cl}
\left(C_{i}(Y)-\overline{C_{l}(Y)}\right) \times \frac{W_{c i}}{W_{c i}(Y)}+\overline{C_{l}(Y)} & \text { if }\left(Y<K_{i}\right) \text { or }\left(K_{h}<Y\right) \\
C_{i}(Y) & \text { if }\left(Y \in\left[K_{i}, K_{h}\right]\right)
\end{array}\right.
$$

After the segmented color transformation, it is projected onto the two-dimensional subspace to obtain a practical skin color clustering model. In this paper, different color has the same two-dimensional Gaussian model, calculate the similarity of face images in all pixel colors and color according to the Gaussian model of skin color, then the similarity value of each point is used as the gray value, obtain the similarity distribution of the image and the distribution map is two valued.

In some cases, the face candidate region causes false detection, which makes the face location inaccurate. In various facial features, eyes and mouth are the most significant, map of the eyes and mouth to locate, constitute the isosceles triangle center as the origin of the face ellipse template to locate the face, the effect is more significant. Eye mapping is determined by the color mapping and brightness mapping, the two mapping into a map to determine the center of the eye. The color mapping is constructed as:

$$
E_{c}=\frac{1}{3}\left[(C b)^{2}+(\overline{\mathrm{Cr}})^{2}+(C b / C r)\right]
$$


In the formula, $\mathrm{Cb}$ and $\mathrm{Cr}$ are normalized to 0 to 255, $\mathrm{Cr}$ and $\overline{\mathrm{Cr}}$ are complementary relations. The eye brightness mapping is obtained by a hemispherical structure element expansion and corrosion image, it consists of:

$$
E_{l}=\frac{Y(x, y) \oplus g_{a}(x, y)}{Y(x, y) \otimes g_{a}(x, y)+1}
$$

The symbol $\oplus_{\text {and }} \otimes$ in the formula represent the expansion and corrosion operation, $\mathrm{Y}$ (x,y) for the brightness function, $\mathrm{g}_{\mathrm{a}}(\mathrm{x}, \mathrm{y})$ for the hemisphere structure function, the eye color and brightness mapping combined to determine the eye center.

$$
\mathrm{E}=\left(E_{C}\right) \operatorname{and}\left(E_{L}\right)
$$

The structure of mouth mapping is as follows:

$$
\begin{aligned}
& M_{0}=(C r)^{2} \times \theta\left[(C r)^{2}-C r / C b\right]^{2} \\
& \theta=0.95 \frac{\frac{1}{n} \sum_{(x, y) \in Y G}(C r(x, y))^{2}}{\frac{1}{n} \sum_{(x, y) \in Y G} \operatorname{Cr}(x, y) / C b(x, y)}
\end{aligned}
$$

Operate of the center of the eye and the mouth center to get the face feature map, and determine the central point of the connected area, the triangle is constructed by the center points. According to the geometric relationship of the eyes and the mouth, the isosceles triangle template is get, the center of gravity is the origin of the ellipse, and the face is calibrated by the ellipse ${ }^{[5]}$. It has a good effect on the orientation of the face and the face rotation after a certain angle, According to the detected face region, the regional image of the face above can be obtained.

\section{Feature extraction}

Helmet feature selection is the key factor of safety cap recognition, the color of the national grid system helmet is a distinction, all units use the helmet is divided into red, orange, blue and white ${ }^{[6]}$, according to the different working environment, the helmet color is also different, considering some people may wear the other hat or scarf etc., we select color feature combined with the characteristic shape of the method to judge whether to wear a safety helmet.

Because the color histogram of the image has scale invariance and rotation invariance, the color histogram is the most commonly used method to express the color feature ${ }^{[7-8]}$. Color histogram is a function of the color information, which represents the image with the number of levels of the same color pixels, On the basis of a given color space, the number of pixels of each color component accounted for the proportion of the total number of pixels of the image, so as to get the image of the proportion of the color component histogram. Let A (x, y) is a color image of RGB space, and its color histogram is defined as:

$$
\begin{aligned}
& \mathrm{H}_{A}(k)=\sum_{x=0}^{M-1} \sum_{y=0}^{N-1} h(A(x, y)) \quad k=0,1, \ldots \ldots k
\end{aligned}
$$

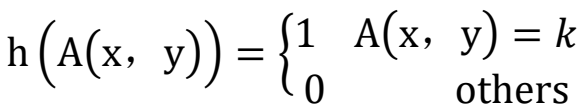

$\mathrm{M}, \mathrm{N}$ is the number of pixels in the vertical and horizontal direction of the image $\mathrm{A}(\mathrm{x}, \mathrm{y})$, referred to as the image of high and wide, while the $\mathrm{K}$ is the color of the transformation space. First, the 3 x 3 neighborhood of each pixel is used to replace the color value of the current pixel with the most color in the neighborhood; Then each pixel statistics histogram, the histogram values of the largest color value as the color of the hat area, if it's black that he don't distribution safety helmet, otherwise combine the shape feature extraction to confirm whether as a safety helmet.

There are two ways to represent the shape feature, one is the profile feature, the other is the regional feature. 
Contour based shape feature extraction method is to obtain the shape of the contour through the boundary pixels of the target image, without taking into account the connection and the relationship of the internal pixel points ${ }^{[9-10]}$. We select the perimeter and the diameter to describe of the helmet, A contour line chain code is a number of pixels of even number is $\mathrm{M}$, the number of pixels of odd number is $\mathrm{N}$, the perimeter of the connected area is:

$$
\mathrm{P}=\mathrm{M}+\sqrt{2} N
$$

Diameter as:

$$
\mathrm{D}=\max _{i j}\left[D_{d}\left(b_{i}, b_{j}\right)\right]
$$

$\mathrm{b}_{\mathrm{i}}$ and $b_{j}$ are the point of the contour, $\mathrm{D}_{\mathrm{d}}$ for any kind of distance, different measures of the corresponding $\mathrm{D}$ value is also different.

\section{Helmet recognition}

By extracting the color features and color features, combined with Canny operator to identify the feature matching, the recognition results are shown in figure 2. From the chart we can see that when staff go through our surveillance area, the system can accurately identify whether to wear a helmet, and automatically display the alarm information, while third figure shows that the situation can be excluded from other hats.
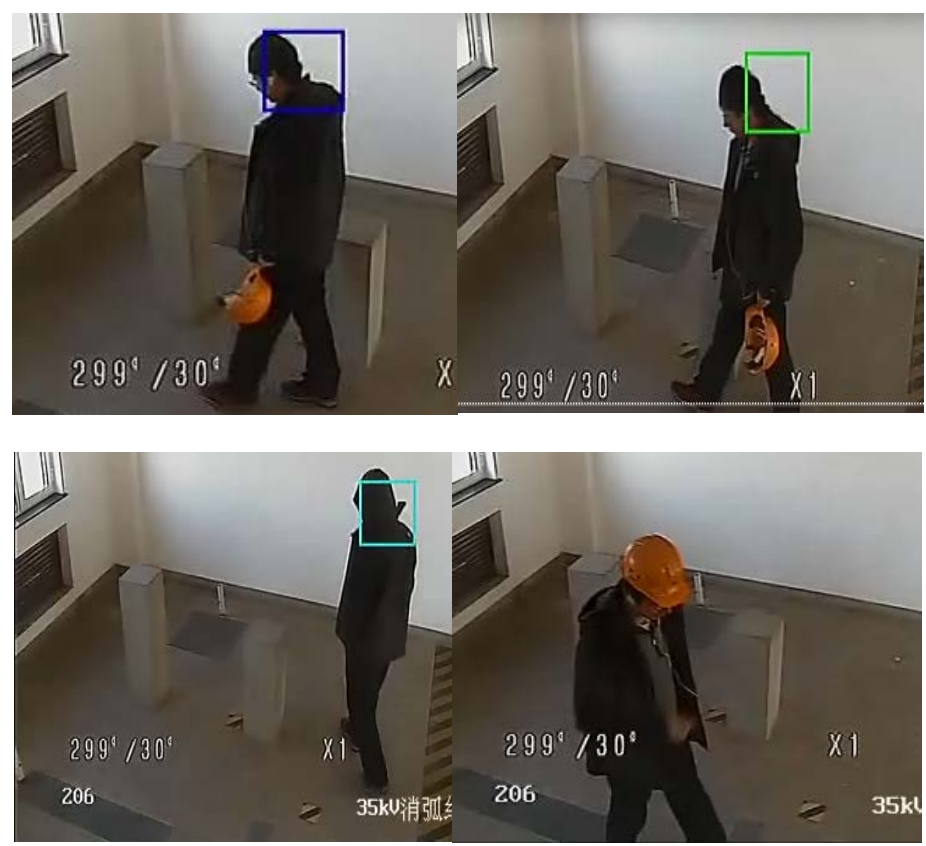

Figure 2. Recognition results

\section{Conclusion}

For substation complex and dangerous work environment, we combine the video recognition technology, puts forward a based on color and shape features of the helmet recognition method, At first, the face of the staff in the monitoring area is located, and then the area above the face is scanned, and the color feature and shape feature are extracted to judge .The method of real-time video acquisition and verification based on safety helmet is more intuitive, with a higher rate through the verification, recognition accuracy rate is above $90 \%$.The method is based on the substation environment, without deliberately artificial, flexible with all kinds of different focal lengths of the camera to complete the verification, and have a higher pass rate.

\section{Acknowledgement}

This study was supported by Research on the Application of Video Recognition Technology in Power Grid 
(XX83-15-001).

\section{References}

[1] Cai Limei. Research on target detection and tracking of people under the coal mine based on video [D]. Xuzhou: China University of Mining and Technology, 2010.

[2] Zhou Yi. Research on key technologies of low resolution faces image recognition [D]. Chengdu: University of Electronic Science and technology of China, 2011.

[3] Huang Chuzhi. Application of intelligent video analysis safety cap identification algorithm in electric power [J].Automation applications, 2015

[4] Shen Changyu. Research on human faces localization by skin color modeling and skin color segmentation [J]. Photoelectric engineering, 2007 (11).

[5] Liu Xiaohui. Application of skin color detection and Hu moments in the identification of safety cap [J]. Journal of east china university of science and technology (NATURAL SCIENCE EDITION), 2014.

[6] Qu Yanhui. Research on image retrieval based on color feature [J]. Computer software and application, 2011, 23 (18):176-179.

[7] Li Taihua. Staff identity authentication algorithm based on color recognition of safety helmet in the transformer substation applications [J]. Computer knowledge and technology, 2014.

[8] Gong Yanhua. Color feature extraction based on color histogram [J]. Fujian computer, 2007.

[9] Ge Jie. Research on progress of image shapes feature extraction method based on machine vision [J]. Journal of packaging, 2015.

[10] Wang chi-lin. Digital image shape feature extraction research [J]. Journal of microelectronics and computers, 2010.

[11] Liu Yunbo. Monitoring technologies of construction site safety helmet wearing condition monitoring technology [J]. Electronic Science and technology, 2015.

\section{Author profile:}

Zhang Geng (1983-), male, senior engineer, the main research direction for intelligent video analysis, energy Internet, Internet of things, etc..

Li Dan (1992), female, master's graduate student, the main research direction for computer vision and pattern recognition and intelligent video analysis in the power grid application. 